\title{
Hydrothermal Preparation and Characterization of Ultralong Strontium-Substituted Hydroxyapatite Whiskers Using Acetamide as Homogeneous Precipitation Reagent
}

\author{
Jianqiang Xu, Yaoqi Yang, Rong Wan, Yuhui Shen, and Weibin Zhang \\ Department of Orthopaedics, Shanghai Institute of Orthopaedics and Traumatology, Shanghai Ruijin Hospital, \\ Shanghai Jiaotong University School of Medicine, 197 Ruijin Er Road, Shanghai 200025, China
}

Correspondence should be addressed to Yuhui Shen; yuhuiss@163.com and Weibin Zhang; weibin@medmail.com.cn

Received 26 August 2013; Accepted 24 November 2013; Published 28 January 2014

Academic Editors: B. Li and E. Odermatt

Copyright (C) 2014 Jianqiang Xu et al. This is an open access article distributed under the Creative Commons Attribution License, which permits unrestricted use, distribution, and reproduction in any medium, provided the original work is properly cited.

\begin{abstract}
The ultralong strontium- (Sr-) substituted hydroxyapatite (SrHAp) whiskers were successfully prepared using acetamide as homogeneous precipitation reagent. The effect of the Sr substitution amount on the lattice constants and proliferation of human osteoblast cells (MG-63) was further investigated. The results showed that the SrHAp whiskers with diameter of 0.2-12 $\mu \mathrm{m}$ and ultralong length up to $200 \mu \mathrm{m}$ were obtained and the Sr substitution level could be facilely tailored by regulating the initial molar ratio of $\mathrm{Sr} /(\mathrm{Sr}+\mathrm{Ca})$ in raw materials. The $\mathrm{Sr}^{2+}$ replaced part of $\mathrm{Ca}^{2+}$ and the lattice constants increased apparently with the increase of the Sr substitution amount. Compared with the pure HAp whiskers, the Sr substitution apparently stimulated the proliferation of MG-63 at certain extracted concentrations. Our study suggested that the obtained SrHAp whiskers might be used as bioactive and mechanical reinforcement materials for hard tissue regeneration applications.
\end{abstract}

\section{Introduction}

The hydroxyapatite $\left[\mathrm{Ca}_{10}\left(\mathrm{PO}_{4}\right)_{6}(\mathrm{OH})_{2}\right.$, HAp] bioceramics are common bone graft materials and are widely used in biomedical fields due to their excellent biocompatibility, osteoconductive properties, and similarity to the inorganic component of natural bones and teeth [1-3]. However, the mechanical property of HAp materials is unsatisfactory, especially for the toughness, which has severely hindered their wider clinical applications $[4,5]$. Moreover, the HAp bioceramics are largely considered to be lacking in the osteoinductive ability, which may impact repair capacity for large bone defects, nonunions, and follow-up function restoration [6]. So far, many strategies have been developed to solve these problems, such as using $\mathrm{ZrO}_{2}$ metals and carbon nanotubes. as mechanical reinforcement $[5,7,8]$. However, these kinds of reinforcements are bioinert and/or nonbiocompatible, which might reduce the bioactivity and biocompatibility of the implants. It is realized that the HAp whiskers might possess great prospect to be used as the mechanical reinforcements because of their excellent biocompatibility [4, 9-15]. It is considered that the traditional HAp whiskers are also lacking in the ability to stimulate the formation of new bone. It is well known that, as a trace element in human body, the strontium (Sr) plays distinctly dual roles in stimulating bone formation and inhibiting bone resorption $[2,16,17]$. The mechanism is thought to lie in $\mathrm{Sr}^{2+}$ ions, which not only can apparently promote osteoblastrelated gene expression and the alkaline phosphatase (ALP) activity of mesenchymal stem cells (MSCs) but also can decrease the differentiation of osteoclasts [18]. As a newly developed drug to prevent osteoporosis, the $\mathrm{Sr}$ ranelate has been confirmed to possess dual effects of stimulating osteoblast differentiation and inhibiting osteoclast activity and bone resorption, and ultimately reduce the incidence of fractures in osteoporotic patients [18, 19]. Moreover, the partial substitution of $\mathrm{Ca}$ by $\mathrm{Sr}$ can apparently improve the biological properties of phosphate and silicate bioceramics and bioglasses $[16,17,20]$. Therefore, compared with the traditional HAp whiskers, the Sr-substituted HAp whiskers (SrHAp) might possess excellent mechanical and biological properties. 
The previous study suggested that the hydrolysis rate of acetamide was apparently lower than that of the traditional urea additive in the hydrothermal homogeneous precipitation method, which might be of great benefit to the rapid growth of whiskers at a low supersaturation $[14,15,21]$. In this study, the ultralong SrHAp whiskers were hydrothermally prepared using acetamide as homogeneous precipitation reagent. Then the effect of $\mathrm{Sr}$ substitution on morphologies, phases, lattice constants, and osteoblast proliferation of the products was further studied.

\section{Materials and Methods}

2.1. Synthesis and Characterization of SrHAp Whiskers. The SrHAp whiskers designed $\mathrm{Sr} /(\mathrm{Ca}+\mathrm{Sr})$ molar ratios of $0.025,0.05$, and 0.1 were hydrothermally synthesized using acetamide as homogeneous precipitation reagent. Aqueous solutions containing $50 \mathrm{mmol}\left(\mathrm{Ca}^{2+}+\mathrm{Sr}^{2+}\right)$ ions and 29.94 mmol $\mathrm{HPO}_{4}{ }^{2-}$ were prepared by dissolving analytical grade reagents of $\mathrm{Ca}\left(\mathrm{NO}_{3}\right)_{2} \cdot 4 \mathrm{H}_{2} \mathrm{O}, \mathrm{Sr}\left(\mathrm{NO}_{3}\right)_{2}$, and $\mathrm{NH}_{4} \mathrm{H}_{2} \mathrm{PO}_{4}$ in distilled water with $1 \mathrm{~mol} / \mathrm{L}$ acetamide. The $0.1 \mathrm{~mol} / \mathrm{L} \mathrm{HNO}_{3}$ solution was used to adjust the $\mathrm{pH}$ to around 2.75 to obtain clear solutions. Then $85 \mathrm{~mL}$ of the obtained solution was transferred into $100 \mathrm{~mL}$ Teflon autoclaves and heated at $180^{\circ} \mathrm{C}$ for $10 \mathrm{~h}$, followed by cooling to room temperature naturally. After hydrothermal reaction, the obtained suspensions were filtrated and washed with distilled water and anhydrous ethanol for 3 times, respectively, and then dried at $120^{\circ} \mathrm{C}$ for $24 \mathrm{~h}$. The pure HAp whiskers in the absence of Sr substitution were prepared as the control sample via the similar method.

The obtained products were characterized by X-ray diffraction (XRD: D/max 2550 V, Rigaku, Japan) with monochromated $\mathrm{Cu}-\mathrm{K} \alpha$ radiation. The lattice constants were calculated from the well-determined positions of the intense XRD diffractions that were processed by MDI Jade 6.1 software (Materials Data Inc., USA) [22]. The whiskers were also characterized using the Fourier transform infrared spectroscopy (FTIR: Nicolet Co., USA). The morphology and size of the whiskers were observed on field emission scanning electron microscopy (FESEM: JSM-6700F, JEOL, Japan), and the chemical compositions of the powders were analyzed by inductively coupled plasma atomic emission spectroscopy (ICP-AES; VISTA AX, Varian Co., USA) after dissolving the whiskers in $0.1 \mathrm{~mol} / \mathrm{L}$ hydrochloric acid aqueous solution.

2.2. Effect of Ionic Products from SrHAp Whiskers on MG63 Proliferation. The ionic extract method is a widely used international standard to evaluate the effect of the chemical compositions on cell biological responses, which can effectively avoid the extra effects that came from the material morphologies via directly incubating the materials with cells $[2,23]$. Herein, the human osteoblast cells (MG-63, Cell bank, Shanghai, China) were cultured in the medium consisting of a-MEM (89\%, GIBCO, Invitrogen, Grand Island, NY, USA), fetal bovine serum (10\%, FBS; Gibco, USA), and penicillin streptomycin (1\%, PS; Gibco, USA). To prepare the extracts, a stock solution of $50 \mathrm{mg} / \mathrm{mL}$ was first prepared by adding the whiskers into DMEM (GIBCO Invitrogen, Grand Island,
NY) culture medium. After incubation at $37^{\circ} \mathrm{C}$ for $24 \mathrm{~h}$, the mixtures were centrifuged and the supernatants were collected. The serial diluted extracts (25 and $12.5 \mathrm{mg} / \mathrm{mL}$ ) were prepared by diluting the stock solutions with serumfree DMEM. Subsequently, these extracts were sterilized by filtration through $0.2 \mu \mathrm{m}$ filter membranes for cell culture experiments. The ion concentrations of the extracts were measured by ICP-AES.

The MG-63 was seeded in 96-well plates at a density of $5 \times 10^{3}$ cell/well and cultured by incubation at $37^{\circ} \mathrm{C}$ for 5 days with $5 \% \mathrm{CO}_{2}$ and $95 \%$ air at $100 \% \mathrm{RH}$. The medium in the well was then replaced by the prepared extracts. The culture medium was changed every 2 days. After 5 days of culture, $10 \mu \mathrm{L}$ (5 mg/mL) of 3-(4,5-dimethylthiazol-2-yl)-2,5diphenyl tetrazolium bromide (MTT, Dojindo, Kumamoto, Japan) plus $100 \mu \mathrm{L}$ of DMEM were added into each well. After additional incubation for $4 \mathrm{~h}$, the MTT solution was removed and replaced with $100 \mu \mathrm{L}$ of dimethylsulfoxide (DMSO). After 10 min of slow shaking (Vibramax 100, Metrohm, USA), the absorbance was read at $570 \mathrm{~nm}$ against the reference value at $630 \mathrm{~nm}$, and the results were expressed as optical density (OD). All experiments were done in triplicate to obtain the average data.

2.3. Statistical Analysis. Data were analyzed for statistical significance using an analysis of variance. Differences at $P$ values of $<0.05$ were considered significant.

\section{Results and Discussion}

3.1. Characterization of SrHAp Whiskers. Figure 1 shows the morphologies of the obtained products. It is clear to see that all of the products were whisker-like morphology with diameter of $0.2-12 \mu \mathrm{m}$ and ultralong length up to $200 \mu \mathrm{m}$. Almost none of the particles were observed. The SEM observation suggested that the Sr substitution did not alter the morphologies of the products. In the homogeneous precipitation method, the reagent of urea is usually applied as the additive to raise the $\mathrm{pH}$ value to drive the nucleation and growth of HAp crystals under hydrothermal treatment $[1,24]$. Compared with the additive of urea, the acetamide possesses a lower hydrolysis rate under the required hydrothermal conditions, which allows better and easier control and gives rise to rapid growth of whiskers at low supersaturation [21]. Moreover, the average of the diameters and lengths of the SrHAp whiskers were almost $1.47-1.85$ and $0.46-0.61$ times higher than those of the HAp whiskers, respectively. The results suggested that the Sr-substitution might increase the size of the whiskers. However, the mechanism needs to be further investigated in detail.

Figure 2 presents the XRD patterns of the obtained HAp and Sr-substituted HAp whiskers. The results showed that all of the products could be well identified as pure HAp phase (JCPDS card: NO. 09-0432). Compared with the pure HAp whiskers, the small angle XRD scanning results (Figure 2(b)) clearly showed that the corresponding peaks of the obtained Sr-substituted HAp whiskers shifted to lower degree. Moreover, with the increase of the Sr substitution level, the shifting 
TABLE 1: Lattice constants, $2 \theta$ value for (300) diffraction, chemical composition, and size of the synthetic HAp and Sr-substituted HAp whiskers.

\begin{tabular}{|c|c|c|c|c|c|c|c|}
\hline \multirow[b]{2}{*}{ Samples } & \multicolumn{2}{|c|}{ Lattice constants } & \multirow{2}{*}{$\begin{array}{l}2 \theta\left(^{\circ}\right) \text { for }\left(\begin{array}{lll}3 & 0 & 0\end{array}\right) \\
\text { reflection }\end{array}$} & \multicolumn{2}{|c|}{ Chemical composition } & \multicolumn{2}{|c|}{ Size of the whiskers } \\
\hline & $a(\AA)$ & $c(\AA)$ & & $\begin{array}{l}\text { Ca replacement } \\
\text { by Sr (mol.\%) }\end{array}$ & $\begin{array}{l}(\mathrm{Ca}+\mathrm{Sr}) / \mathrm{P} \\
\text { moler ratio }\end{array}$ & Length $(\mu \mathrm{m})$ & Diameter $(\mu \mathrm{m})$ \\
\hline HAp & 9.423 & 6.915 & 32.900 & 0 & 1.62 & $33.64 \pm 19.10$ & $2.19 \pm 1.84$ \\
\hline Sr2.5HAp & 9.439 & 6.883 & 32.841 & 1.37 & 1.65 & $83.09 \pm 46.24$ & $3.21 \pm 1.58$ \\
\hline Sr5HAp & 9.445 & 6.912 & 32.821 & 2.72 & 1.63 & $86.36 \pm 43.28$ & $3.53 \pm 1.37$ \\
\hline Sr10HAp & 9.451 & 6.964 & 32.800 & 5.83 & 1.61 & $95.76 \pm 37.65$ & $3.45 \pm 1.26$ \\
\hline
\end{tabular}

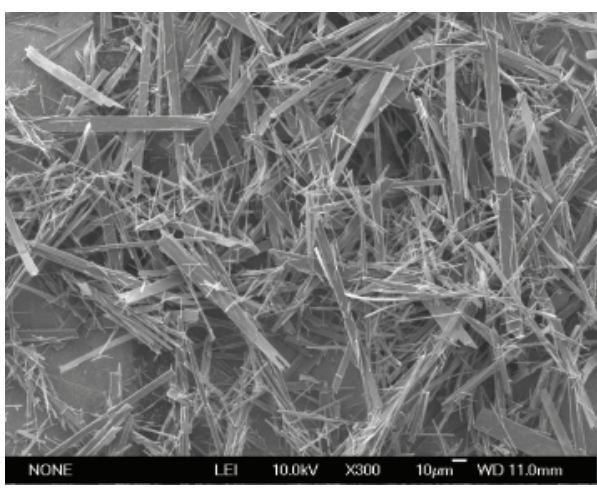

(a)

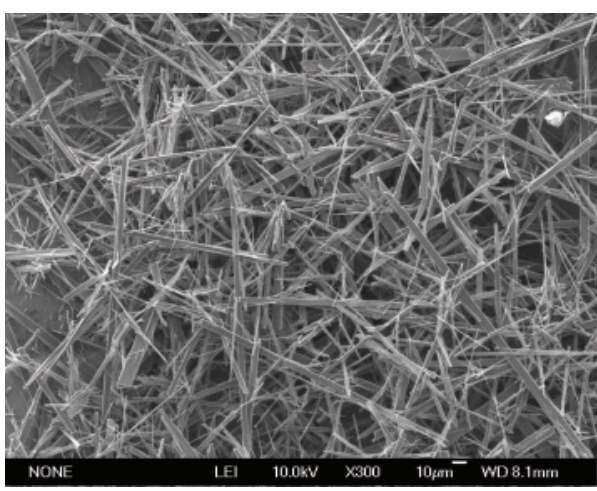

(c)

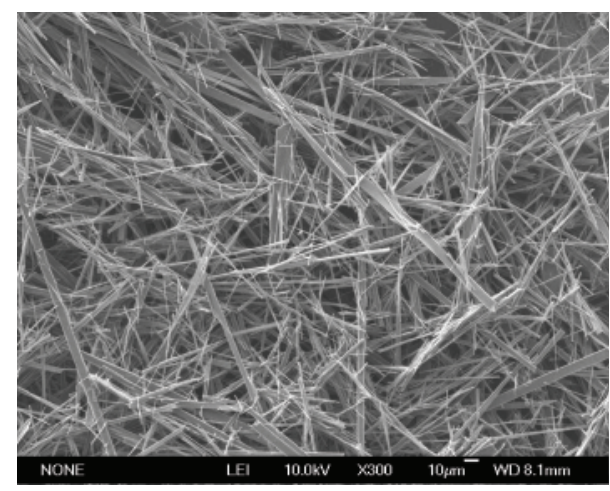

(b)

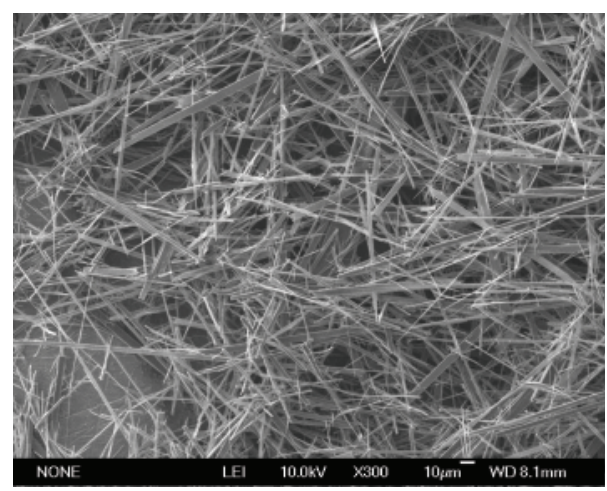

(d)

Figure 1: SEM images of the obtained HAp (a), Sr2.5HAp (b), Sr5HAp (c), and Sr10HAp (d) whiskers; Bar $=30 \mu \mathrm{m}$.

extent increased apparently, suggesting the increase of the lattice constants (Table 1) [2]. The calculated lattice constants based on the XRD determination results validated that the lattice constants of the prepared Sr-substituted HAp whiskers were larger than those of the pure HAp whiskers (Table 1). The increase of the lattice constants was attributed to the replacement of the $\mathrm{Ca}^{2+}$ ions by bigger diameter of $\mathrm{Sr}^{2+}$ ions [2]. The shifting of the patterns and the deviation of lattice constants suggested that the $\mathrm{Sr}^{2+}$ ions replaced and occupied the $\mathrm{Ca}^{2+}$ crystal positions of the HAp $[2,3]$. However, the peak intensities of the $\left(\begin{array}{lll}2 & 1 & 1\end{array}\right)$ and $\left(\begin{array}{lll}3 & 0 & 0\end{array}\right)$ reflections were different from the standard values, which was attributed to the preferential orientation growth of the HAp whiskers.

Figure 3 reveals the FTIR spectra of the obtained HAp and Sr-substituted HAp whiskers. The spectra accord well with the reported FTIR data for HAp. The peaks at 473,
$563,603,962,1031$, and $1095 \mathrm{~cm}^{-1}$ were the characteristic bands for $\mathrm{PO}_{4}{ }^{3-}[1-3]$. The peaks at 3442 and $1637 \mathrm{~cm}^{-1}$ were assigned to the bending mode of the absorbed water. The peak at $868 \mathrm{~cm}^{-1}$ was attributed to the carbonate ion in the B-site, which might come from the dissolved carbon dioxide in aqueous solution [16]. The peaks at 3570 and $633 \mathrm{~cm}^{-1}$ were the characteristic $\mathrm{OH}$ bands of HAp [3]. The FTIR results further confirmed that the positions of the peaks were not affected by Sr substitution and the products were HAp crystals.

The ICP-AES analytic results of the obtained whiskers are presented in Table 1 . The results showed that the prepared SrHAp whiskers contained the substituted ions of $\mathrm{Sr}$ and the substitution amount of Sr in the obtained Sr-HAp whiskers increased obviously with the increase of the initial molar ratio of $\mathrm{Sr} /(\mathrm{Sr}+\mathrm{Ca})$ in raw materials. In addition, the $(\mathrm{Ca}+\mathrm{Sr}) / \mathrm{P}$ 


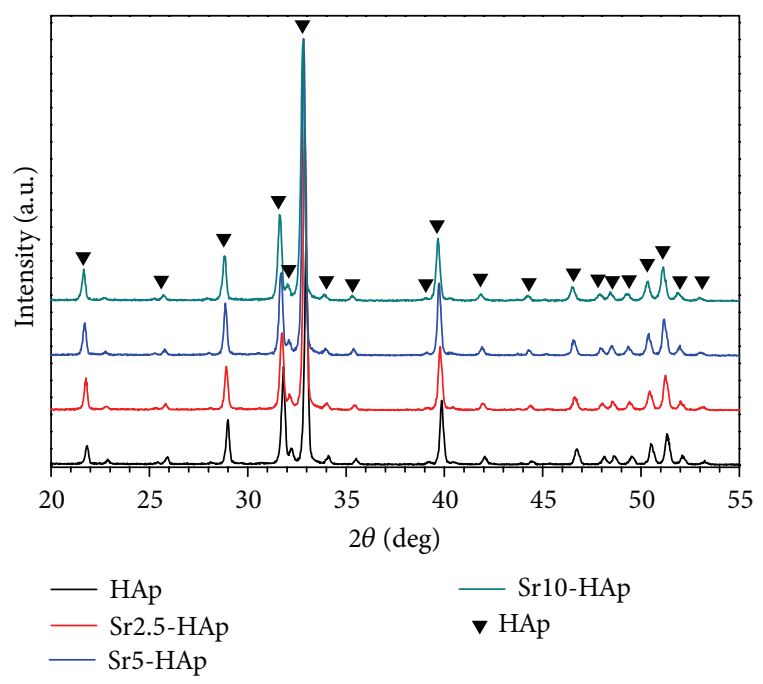

(a)

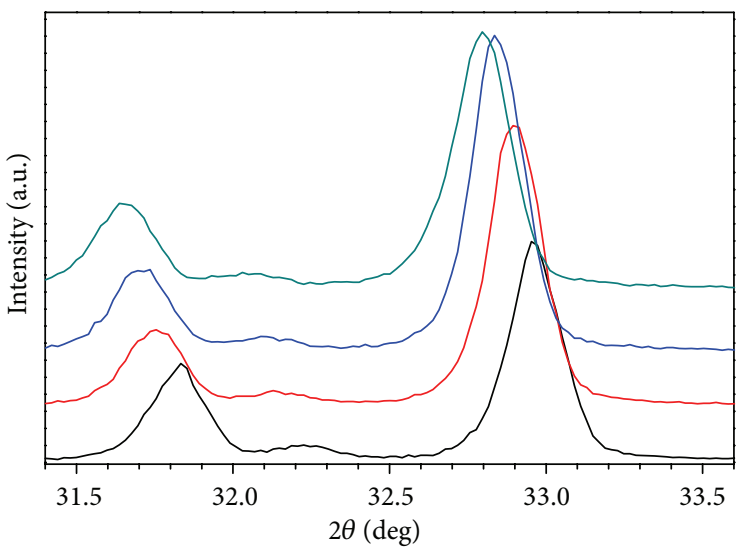

(b)

FIGURE 2: XRD patterns of the obtained HAp and Sr-substituted HAp whiskers.

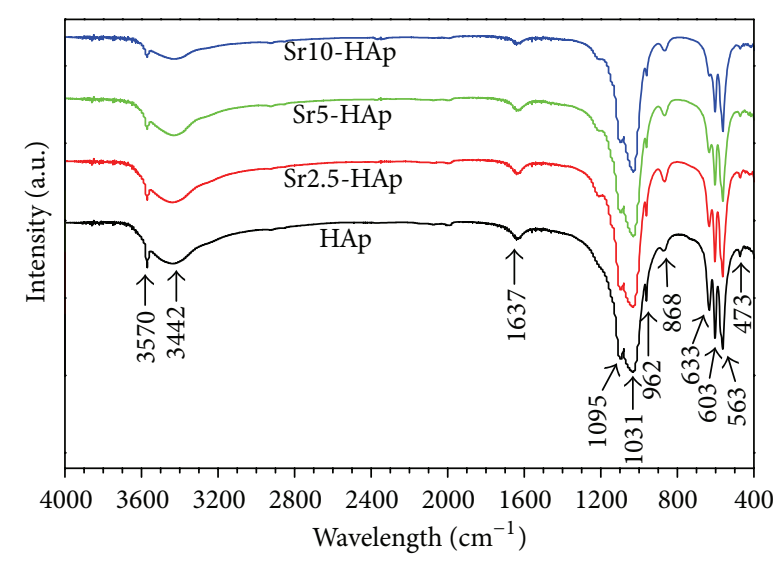

FIGURE 3: The FTIR spectra of the obtained HAp and Sr-substituted HAp whiskers.

molar ratio of the obtained Sr-substituted HAp whiskers was between 1.61 and 1.65, which was slightly deviated from the stoichiometric HAp $(\mathrm{Ca} / \mathrm{P}=1.67)$.

3.2. Effect of Ionic Products from SrHAp Whiskers on MG63 Proliferation. Compared with pure HAp, a stimulatory effect of the ionic products from Sr-substituted HAp whiskers at appropriate concentrations on MG-63 proliferation was observed (Figure 4). The stimulatory effects of the ionic product from Sr5-HAp whiskers on MG-63 proliferation were apparently higher than other materials between the extract concentrations of 12.5 and $50 \mathrm{mg} / \mathrm{mL}$. The results indicated the better therapeutic potential of Sr5-HAp whiskers for bone regeneration. Previous studies suggested that the effects of Sr on bone cells were dose-dependent [25]. The ICP-OES analysis showed that the $\mathrm{Sr}$ ion concentrations of the Sr5HAp whisker extracts $(12.5-50 \mathrm{mg} / \mathrm{mL})$ for cell culture were $2.88 \times 10^{-3}-1.15 \times 10^{-2} \mathrm{mmol} / \mathrm{L}$. The study of Bonnelye et al. confirmed that $\mathrm{Sr}$ ranelate with $\mathrm{Sr}^{2+}$ concentrations around

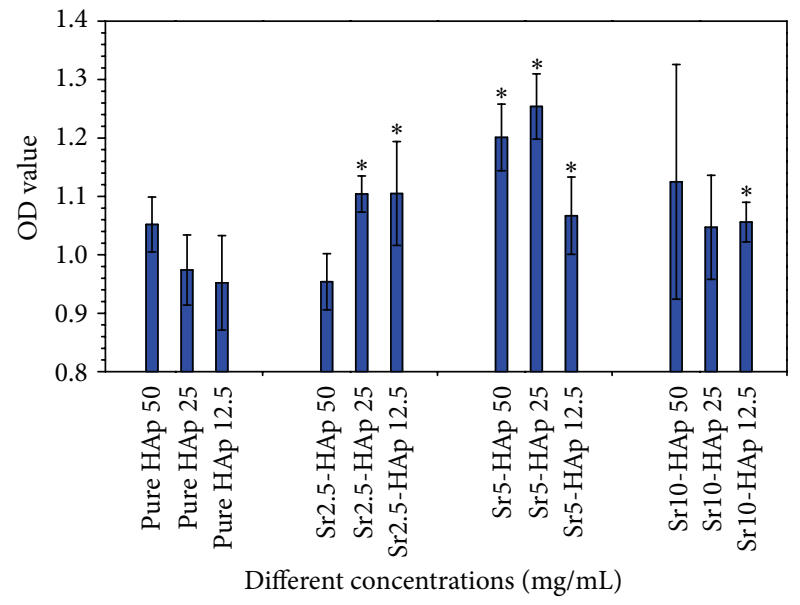

FIGURE 4: The effect of ionic products from HAp and Sr-substituted HAp whiskers on proliferation of MG-63 after 5 days of culture. * indicates the experimental group compared with the control group of HAp whiskers at the same concentration, ${ }^{*} P<0.05$.

0.1-1 mmol/L could promote osteoblast formation [26]. In addition, the concentration of $\mathrm{Sr}$ in normal serum varied between $1.14 \times 10^{-4}$ and $2.48 \times 10^{-3} \mathrm{mmol} / \mathrm{L}$ [27], which was much lower than those in our study and the reported references. Moreover, the amount of $\mathrm{Sr}$ in bone tissue is apparently higher than that in blood, and almost $99 \%$ of the absorbed $\mathrm{Sr}$ is deposited in bone $(36-140 \mathrm{mg} / \mathrm{kg})[25,28]$. Therefore, it can be indicated that a certain high dose of $\mathrm{Sr}$ may stimulate the proliferation of osteoprogenitor cells and benefit new bone regeneration [25].

\section{Conclusions}

In this study, the ultralong SrHAp whiskers with 0-5.83 mol $\%$ of Ca substituted by Sr and length up to $200 \mu \mathrm{m}$ have been 
successfully hydrothermally prepared using acetamide as homogeneous precipitation reagent. The Sr substitution level of the whiskers could be well facilely regulated by tailoring the initial molar ratio of $\mathrm{Sr} /(\mathrm{Sr}+\mathrm{Ca})$ in the raw materials. In addition, the $\mathrm{Sr}^{2+}$ ions replaced part of $\mathrm{Ca}^{2+}$ ions, and the lattice constants increased apparently with the increase of the Sr substitution amount. The cell culture results showed that the ionic products of SrHAp whiskers apparently promoted the proliferation of MG-63 at certain concentrations of $\mathrm{Sr}^{2+}$ ions. Particularly the Sr5-HAp component was optimal for cell activity. Our study suggests that the Sr-substituted HAp whiskers might be a potential candidate as a new bioactive material in biomedical field.

\section{Conflict of Interests}

The authors declare that there is no conflict of interests regarding the publication of this paper.

\section{Authors' Contribution}

Jianqiang $\mathrm{Xu}$ and Yaoqi Yang contributed equally to this paper.

\section{Acknowledgments}

The authors gratefully acknowledge the support of the Natural Science Foundation of China (Grant nos. 81371937 and 81150007), Shanghai Municipal Education Commission (Grant no. 12YZ039), Science and Technology Commission of Shanghai Municipality (Grant no. 13ZR1437700), and Shanghai Municipal Commission of Health and Family Planning (20134224).

\section{References}

[1] K. Lin, J. Chang, Y. Zhu et al., "A facile one-step surfactant-free and low-temperature hydrothermal method to prepare uniform 3D structured carbonated apatite flowers," Crystal Growth and Design, vol. 9, no. 1, pp. 177-181, 2009.

[2] K. L. Lin, P. Y. Liu, W. B. Zhang et al., "Mesoporous strontium substituted hydroxyapatite microspheres: surfactant-free hydrothermal synthesis, enhanced biological response and sustained drug release," Chemical Engineering Journal, vol. 222, no. 15, pp. 49-59, 2013.

[3] K. Lin, Y. Zhou, Y. Zhou et al., "Biomimetic hydroxyapatite porous microspheres with co-substituted essential trace elements: surfactant-free hydrothermal synthesis, enhanced degradation and drug release," Journal of Materials Chemistry, vol. 21, no. 41, pp. 16558-16565, 2011.

[4] K. Lin, J. Chang, R. Cheng, and M. Ruan, "Hydrothermal microemulsion synthesis of stoichiometric single crystal hydroxyapatite nanorods with mono-dispersion and narrowsize distribution," Materials Letters, vol. 61, no. 8-9, pp. 1683-1687, 2007.

[5] D. Lahiri, S. Ghosh, and A. Agarwal, "Carbon nanotube reinforced hydroxyapatite composite for orthopedic application," Materials Science and Engineering C, vol. 32, no. 7, pp. 1727-1758, 2012.
[6] H. Yuan, H. Fernandes, P. Habibovic et al., "Osteoinductive ceramics as a synthetic alternative to autologous bone grafting," Proceedings of the National Academy of Sciences of the United States of America, vol. 107, no. 31, pp. 13614-13619, 2010.

[7] M. A. F. Afzal, P. Kesarwani, K. M. Reddy, S. Kalmodia, B. Basu, and K. Balani, "Functionally graded hydroxyapatite-aluminazirconia biocomposite: synergy of toughness and biocompatibility," Materials Science and Engineering C, vol. 32, no. 5, pp. 1164-1173, 2012.

[8] M. Mittal, S. K. Nath, and S. Prakash, "Improvement in mechanical properties of plasma sprayed hydroxyapatite coatings by $\mathrm{Al}_{2} \mathrm{O}_{3}$ reinforcement," Materials Science and Engineering C, vol. 33, no. 5, pp. 2838-2845, 2013.

[9] S. Bose, A. Banerjee, S. Dasgupta, and A. Bandyopadhyay, "Synthesis, processing, mechanical, and biological property characterization of hydroxyapatite whisker-reinforced hydroxyapatite composites," Journal of the American Ceramic Society, vol. 92, no. 2, pp. 323-330, 2009.

[10] C. Qi, Q. L. Tang, Y. J. Zhu, X. Y. Zhao, and F. Chen, "Microwaveassisted hydrothermal rapid synthesis of hydroxyapatite nanowires using adenosine $5^{\prime}$-triphosphate disodium salt as phosphorus source," Materials Letters, vol. 85, pp. 71-73, 2012.

[11] X. Wang, J. Zhuang, Q. Peng, and Y. Li, "Liquid-solid-solution synthesis of biomedical hydroxyapatite nanorods," Advanced Materials, vol. 18, no. 15, pp. 2031-2034, 2006.

[12] Y. J. Wang, C. Lai, K. Wei, X. Chen, Y. Ding, and Z. L. Wang, "Investigations on the formation mechanism of hydroxyapatite synthesized by the solvothermal method," Nanotechnology, vol. 17, no. 17, article 020, pp. 4405-4412, 2006.

[13] K. Lin, X. Liu, J. Chang, and Y. Zhu, "Facile synthesis of hydroxyapatite nanoparticles, nanowires and hollow nanostructured microspheres using similar structured hardprecursors," Nanoscale, vol. 3, no. 8, pp. 3052-3055, 2011.

[14] H. Zhang and B. W. Darvell, "Morphology and structural characteristics of hydroxyapatite whiskers: effect of the initial Ca concentration, $\mathrm{Ca} / \mathrm{P}$ ratio and $\mathrm{pH}$," Acta Biomaterialia, vol. 7, no. 7, pp. 2960-2968, 2011.

[15] H. Zhang, Y. Wang, Y. Yan, and S. Li, "Precipitation of biocompatible hydroxyapatite whiskers from moderately acid solution," Ceramics International, vol. 29, no. 4, pp. 413-418, 2003.

[16] K. Lin, J. Chang, X. Liu, L. Chen, and Y. Zhou, "Synthesis of element-substituted hydroxyapatite with controllable morphology and chemical composition using calcium silicate as precursor," CrystEngComm, vol. 13, no. 15, pp. 4850-4855, 2011.

[17] V. Aina, L. Bergandi, G. Lusvardi et al., "Sr-containing hydroxyapatite: morphologies of HA crystals and bioactivity on osteoblast cells," Materials Science and Engineering C, vol. 33, no. 3, pp. 1132-1142, 2013.

[18] S. Peng, G. Zhou, K. D. K. Luk et al., "Strontium promotes osteogenic differentiation of mesenchymal stem cells through the Ras/MAPK signaling pathway," Cellular Physiology and Biochemistry, vol. 23, no. 1-3, pp. 165-174, 2009.

[19] H. B. Pan, X. L. Zhao, X. Zhang et al., "Strontium borate glass: potential biomaterial for bone regeneration," Journal of the Royal Society Interface, vol. 7, no. 48, pp. 1025-1031, 2010.

[20] W. Zhang, Y. Shen, H. Pan et al., "Effects of strontium in modified biomaterials," Acta Biomaterialia, vol. 7, no. 2, pp. 800808, 2011.

[21] H. Zhang and B. W. Darvell, "Synthesis and characterization of hydroxyapatite whiskers by hydrothermal homogeneous precipitation using acetamide," Acta Biomaterialia, vol. 6, no. 8, pp. 3216-3222, 2010. 
[22] S. Koutsopoulos, "Synthesis and characterization of hydroxyapatite crystals: a review study on the analytical methods," Journal of Biomedical Materials Research, vol. 62, no. 4, pp. 600-612, 2002.

[23] "Biological evaluation of medical devices-Part 1. Evaluation and testing within a risk management process," ISO 10993-1, 2009.

[24] S. P. Parthiban, I. Y. Kim, K. Kikuta, and C. Ohtsuki, "Effect of urea on formation of hydroxyapatite through double-step hydrothermal processing," Materials Science and Engineering C, vol. 31, no. 7, pp. 1383-1388, 2011.

[25] M. Zhang, W. Zhai, K. Lin, H. Pan, W. Lu, and J. Chang, "Synthesis, in vitro hydroxyapatite forming ability, and cytocompatibility of strontium silicate powders," Journal of Biomedical Materials Research B, vol. 93, no. 1, pp. 252-257, 2010.

[26] E. Bonnelye, A. Chabadel, F. Saltel, and P. Jurdic, "Dual effect of strontium ranelate: stimulation of osteoblast differentiation and inhibition of osteoclast formation and resorption in vitro," Bone, vol. 42, no. 1, pp. 129-138, 2008.

[27] W. E. Cabrera, I. Schrooten, M. E. De Broe, and P. C. D'Haese, "Strontium and bone," Journal of Bone and Mineral Research, vol. 14, no. 5, pp. 661-668, 1999.

[28] S. C. Skoryna, "Metabolic aspects of the pharmacologic uses of trace elements in human subjects with specific references to stable strontium," Trace Substances in Environmental Health, vol. 18, pp. 3-23, 1984. 

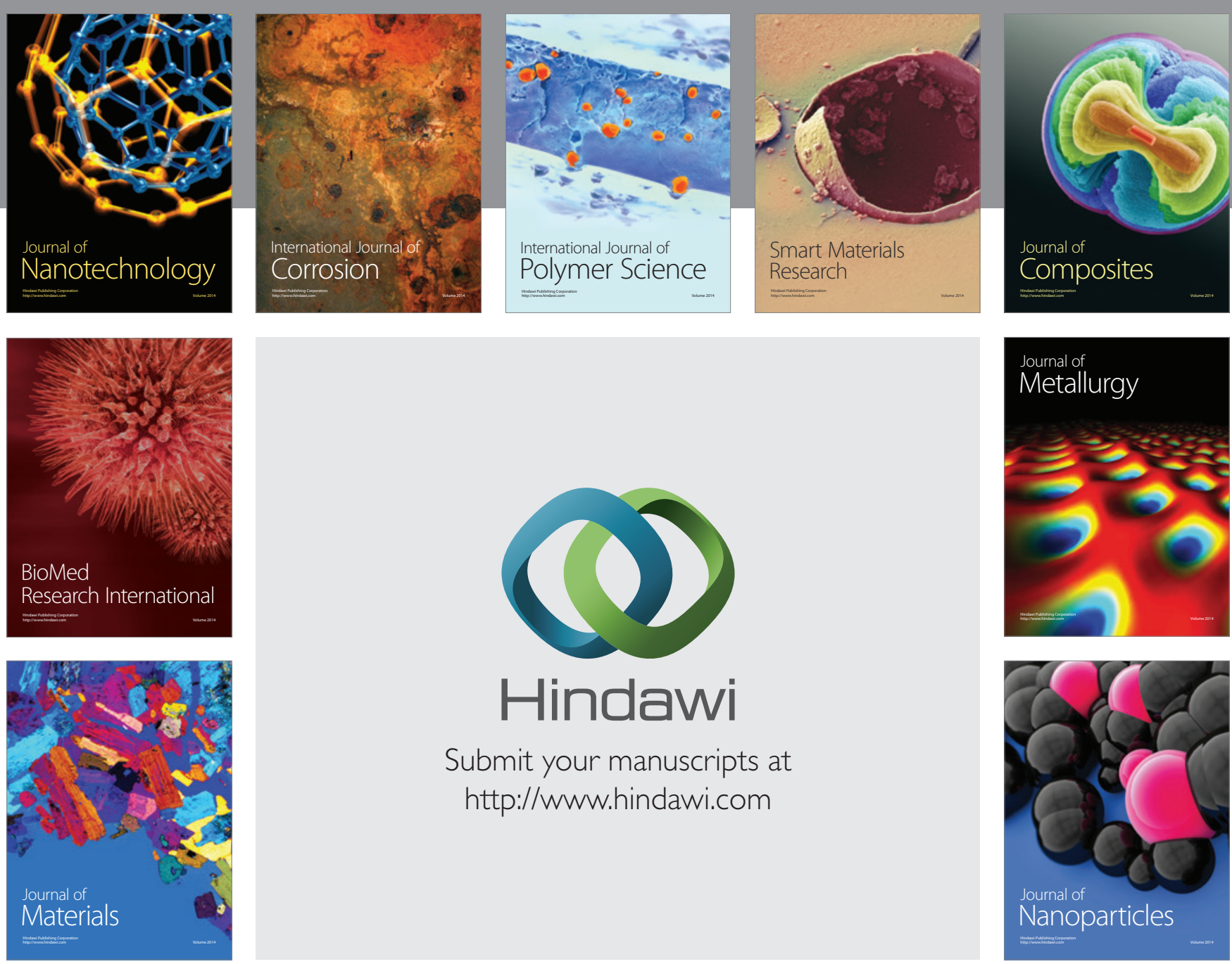

Submit your manuscripts at http://www.hindawi.com
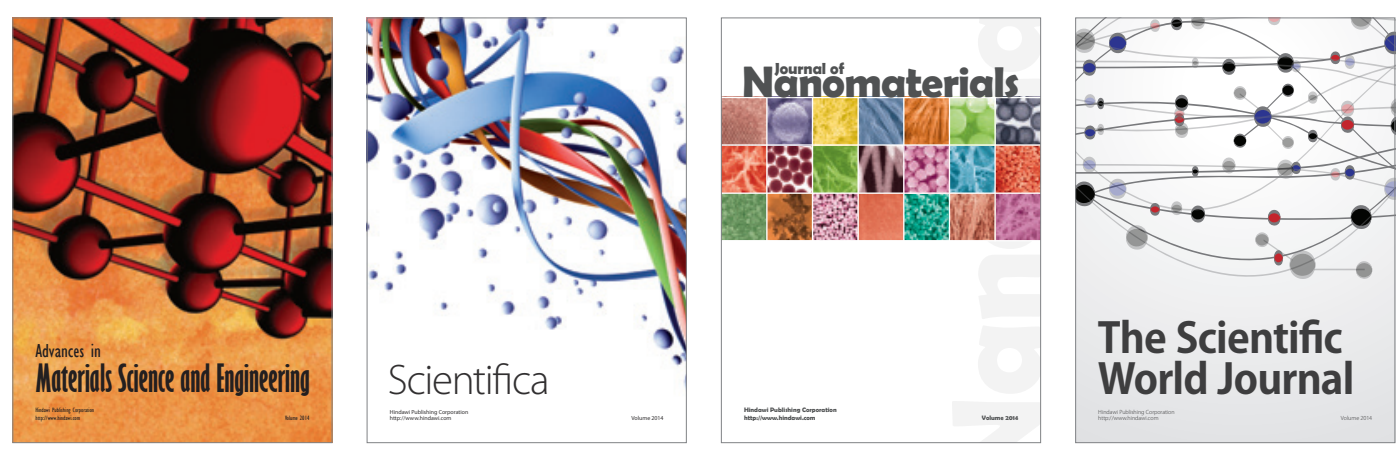

\section{The Scientific World Journal}
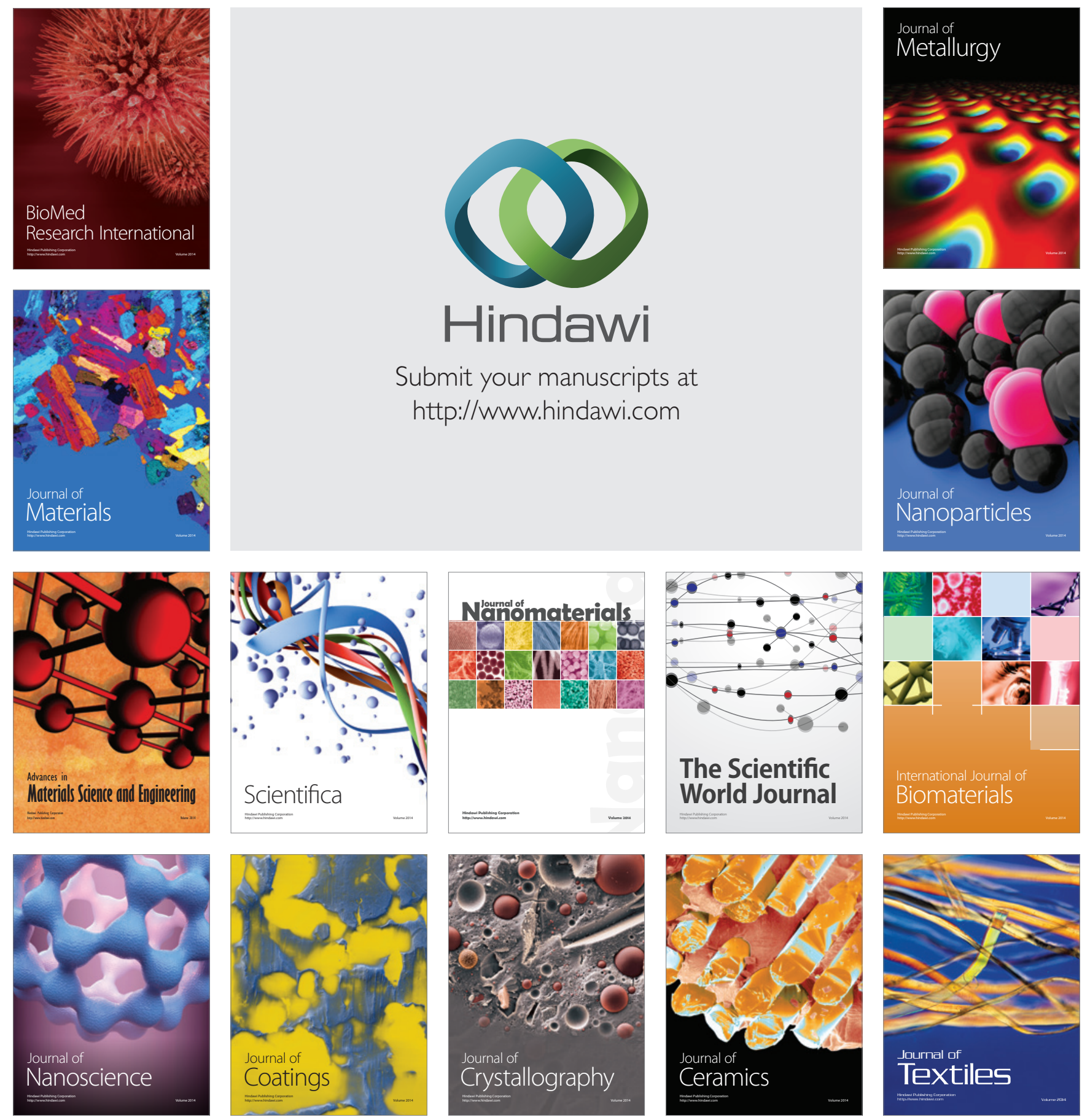